\title{
A discussion of the optimal treatment of intracranial aneurysm rupture in elderly patients
}

\author{
C. Liu
}

Neurology Department, The Central Hospital of Luoyang Affiliated to Zhengzhou University, Luoyang City, Henan Province, China

Corresponding author: C. Liu

E-mail: chaoliucn@126.com

Genet. Mol. Res. 13 (3): 6433-6438 (2014)

Received May 28, 2013

Accepted October 23, 2013

Published August 25, 2014

DOI http://dx.doi.org/10.4238/2014.August.25.6

\begin{abstract}
This study aimed to find an optimal treatment for intracranial aneurysm rupture in elderly patients. We adopted endovascular embolization and combined it with mini-invasive aspiration, vascular stenosis stenting, and rehabilitation training to treat 13 elderly patients with intracranial aneurysm rupture. When the 13 patients were discharged and evaluated by the Glasgow Outcome Score (GOS), 7 patients were grade 5, 4 patients were grade 4 , and 2 patients were grade 2 . We found that a combination of endovascular embolization with mini-invasive aspiration and vascular stenosis stenting allowed us to adapt this treatment to various types of aneurysms. Our approach is especially suitable for elderly patients, because it reduces the occurrence of complications, improves patient prognoses, shortens the duration of hospitalization, and improves the quality of life.
\end{abstract}

Key words: Advanced age; Intracranial aneurysm; Rupture; Optimal treatment 


\section{INTRODUCTION}

Intracranial aneurysm rupture is a dreadful disease condition that results in rapid patient deterioration when untreated. If the disease is not definitively diagnosed and the correct treatment administered, the prognosis will worsen. Standard therapy for intracranial aneurysm rupture associated with hematoma involves conducting a drilling skull operation as soon as possible to clear the intracranial hematoma and clip the aneurysms (Molyneux et al., 2005). However, in elderly patients, the treatment of intracranial aneurysm rupture has its own characteristics: 1) patients frequently have various chronic diseases, such as heart disease or diabetes; 2) the disease becomes worse after onset; 3 ) the disease is frequently associated with cerebral artery stenosis and aneurysm artery tortuosity; and 4) we regularly refer to a subarachnoid hemorrhage as the form of disease outset and associate it with intracerebral hematoma or ventricular hemorrhage. Elderly people tend to bleed profusely in the acute stage, develop worsening brain edema, have high intracranial pressure, and an unclear visual operative field. In addition, drilling skull operations are more prone to cause secondary damage in elderly patients. The postoperative effects of treating elderly patients with intracranial aneurysm rupture are often unsatisfactory and take a long time to resolve. Together with an extended duration of postoperative bed rest, which can cause pulmonary infection, cerebral or myocardial infarction, and a series of other complications, this procedure brings heavy burdens to society and thousands of families.

Clipping the neck of the aneurysm in a drilling skull operation or by interventional embolization can successfully remove the threat of aneurysm (Sugiu et al., 2005), thereby improving the prognosis of the patient. The latest international subarachnoid aneurysm trial (ISAT) developments have shown that the integrated endovascular embolization approach is superior to the clipping operation for elderly patients (Molyneux et al., 2005, 2009; Qureshi et al., 2007). Although open cranium hematoma removal and minimally invasive hematoma removal can both remove the threat of hematoma for a patient, most researchers argue that hematoma should be removed by early, minimally invasive surgery (Hodelin-Tablada, 1997). Thus, intravascular interventional embolization and minimally invasive hematoma removal have the advantages of being minimally invasive and comparatively safer, and are associated with a lower risk of trauma and complications of the lung, heart, and other organs that result from operation and anesthesia.

Since 2007, we have adopted intravascular embolization coupled with minimally invasive removal of hematoma, vascular stenosis stenting, and rehabilitation training. To comprehensively treat 13 elderly patients with intracranial aneurysm rupture, this study organically combined these approaches and assessed its safety, feasibility, and effectiveness. Our approach maximally reduces body trauma in elderly patients, facilitating quicker postoperative recovery, an improved prognosis, a shortened hospitalization period, and a better quality of life.

\section{MATERIAL AND METHODS}

\section{General information}

Our study cohort included 13 patients ( 5 men and 7 women); their age ranged from 62 to 78 years, with a mean of 70 years. This study was conducted in accordance with the 
Declaration of Helsinki and with approval from the Ethics Committee of Henan Province at Luoyang Central Hospital. Written informed consent was obtained from all participants. Our cohort of 13 patients included 3 diabetic patients, 9 hypertensive patients, 5 lacunar infarction patients, and 5 coronary heart disease patients. Initial clinical disease manifestations included headache, vomiting, and disturbance of consciousness. By physical examination, we found 13 patients with meningeal irritation and 5 patients with limb paralysis. According to the Hunt and Hess scale, 3 patients were grade 2, 8 patients were grade 3, and 2 patients were grade 4 cases.

\section{Neurological imaging}

Skull computed tomography $(\mathrm{CT})$ showed subarachnoid hemorrhage in all patients. In 5 patients, this was accompanied by intracerebral hematoma, including 3 patients with hematoma in the lateral fissure region and 2 patients with hematoma in the frontal lobe. Blood loss due to hematoma was $20-45 \mathrm{~mL}$ per patient. All patients were diagnosed with an aneurysm by digital subtraction angiography (DSA) assessment within $24 \mathrm{~h}$ after the onset of illness. There were 3 cases with an aneurysm located in an artery that bifurcated the brain, 2 cases with an aneurysm located in an anterior communicating artery, 2 cases with an aneurysm located in an anterior cerebral artery, and 6 cases with an aneurysm located in a posterior communicating artery. There were 3 cases with a large aneurysm (diameter: $1.5-2.5 \mathrm{~cm}$ ), 1 case with combined unilateral internal carotid artery stenosis and vertebral artery opening stenosis, 8 cases with a medium aneurysm (diameter: $0.7-1.4 \mathrm{~cm}$ ), 2 cases with an aneurysm combined with unilateral vertebral artery opening stenosis, 1 case with unilateral internal carotid artery initial segment stenosis, 2 cases with a small aneurysm (diameter: $<0.6 \mathrm{~cm}$ ), and 1 case with an aneurysm combined with unilateral vertebral artery opening stenosis.

\section{Processing method}

To stabilize the vital signs of the patients and facilitate future assessments, all patients were treated by endovascular embolization within 3 days. For patients with an aneurysm and combined internal carotid artery or vertebral artery stenosis, when they underwent aneurysm vascular embolization, an internal carotid or vertebral artery stent was also implemented. For patients with an aneurysm combined with hematoma, we adopted mini-invasive aspiration. For patients with an aneurysm combined with hydrocephalus, we adopted ventriculo-peritoneal drainage. For stent implantation, we preoperatively inhibited platelet activity by rectal or intranasal administration of $300 \mathrm{mg}$ Plavix. Subsequently, we used a needle to inject 5000 U low-molecular-weight heparin calcium subcutaneously, two times per day for 3 successive days. Drugs that were doubly administered, to avoid drug resistance, should be used simultaneously for 3-6 months, after which the dose should be decreased. Other patients were treated with antibiotics to reduce intracranial pressure, remove vasospasms, and improve circulation. Some patients were assisted later by rehabilitation training.

\section{RESULTS}

In this study cohort, all patients were elderly with a 2-4 grade lesion by Hunt and Hess staging. Some patients could not undergo operation because of hematoma, and were, instead, 
treated with vascular embolization. The patients with aneurysm combined with internal carotid artery vertebral artery stenosis were treated immediately.

For patients with vascular thrombosis of the aneurysm and carotid artery (vertebral artery stenting combined hematoma), minimally invasive surgery was elected. For patients with aneurysms combined with hydrocephalus, ventriculo-peritoneal drainage was also used. By 1-2 months after comprehensive treatment, all patients showed good recovery. In cases of large posterior communicating artery and carotid artery aneurysm, the patients with vertebral artery stenosis survived until 5 years postoperatively, when the patients died of acute myocardial infarction. The 3 patients with medium-sized aneurysms and patients with small aneurysms with carotid or vertebral artery stenosis survived for more than 3 years, and are to date being monitored by follow-up. For 5 cases of aneurysm rupture and hematoma formation that underwent vascular embolization of the aneurysm and minimally invasive surgery, the patients showed stable vital signs after more than a month of rehabilitation training. The muscle strength of the affected limb in these patients improved from a score of 0-4 after rehabilitation, and these patients became self-sufficient according to the Glasgow Outcome Score (GOS) assessment of prognosis (Table 1).

Table 1. Attached list Glasgow Ouctcome Score evaluation results.
\begin{tabular}{lccccc}
\hline GOS score & GOS1score & GOS2score & GOS3score & GOS4score & GOS5score \\
\hline No. of samples & 0 & 0 & 2 & 4 & 7 \\
\hline
\end{tabular}

\section{DISCUSSION}

The current approach for treating ruptured intracranial aneurysms complicated with hematoma holds that a drilling skull operation should be conducted as soon as possible to remove intracranial hematocele while also clipping the aneurysm. This approach aims to achieve 2 therapeutic goals and was once regarded as the gold standard for treating ruptured intracranial aneurysms (Molyneux et al., 2005). Although it is important to improve preoperative examinations, accurately mastering the aneurysm clipping operation is an important way to reduce surgical mortality. However, elderly aneurysm patients cannot have other serious underlying diseases and must be physically able to endure operation. For elderly patients, due to frequent underlying diseases and poor operation endurance, the incidence of various complications is increased after an open skull clipping operation (Ryttlefors et al., 2008), and therefore, conservative treatment is currently often adopted, even though it is associated with a mortality rate of up to $71 \%$ (Hijdra et al., 1988). For patients with ruptured intracranial aneurysms complicated with intracerebral hematoma, the prognosis of conservative treatment is worse, with morality rates ranging from 75 to $100 \%$ (Wheelock et al., 1983; Donauer et al., 1993; Shimoda et al., 1997). For the treatment of elderly people for ruptured intracranial aneurysms, there yet is no better treatment.

By analyzing 278 elderly patients in the ISAT, Ryttlefors et al. (Bradac et al., 2005) found that the prognosis for endovascular embolization was much better than for an open skull clamp operation, and the incidence of epilepsy was also much lower. The latest ISAT developments have demonstrated the superiority of integrated endovascular embolization compared to clipping operations (Qureshi et al., 2007; Molyneux et al., 2005, 2009). Jain et al. (2004), 
reported on early active endovascular embolization treatment and found that the rate of good prognoses was $38 \%$. Others have also reported that the curative effect of endovascular treatment is good, trauma is minimal, direct brain tissue injuries can be avoided, and complication rates are low (Redekop, 2006; Suzuki et al., 2006).

Studies of Lubicz et al. $(2004,2005)$ and Linfante and Wakhloo (2007) suggest that endovascular treatment almost completely overlaps with the indications of drilling skull operation. For a high-grade aneurysm, the operation should be conducted early to improve patient prognosis (Qureshi et al., 2007), and to prevent recurrent hemorrhage (Bracard et al., 2002). For intracranial hematoma, most of researchers advocate early, minimally invasive surgery to remove hematoma (Hodelin-Tablada, 1997). Drilling skull operations have a bad effect on brain tissue and overall physical health, so this type of injury, to a large extent, counteracts the advantage of complete craniotomy for the clearance of hematoma. As heavy bleeding can occur in the acute stage, brain edema is often severe, intracranial pressure is generally high, and the visual operative field is frequently vague, the drilling skull operation is prone to cause secondary damage. Especially for patients with intracranial aneurysms complicated with intracranial and extracranial arterial stenosis, anesthesia time is relatively long for drilling skull operation, blood volume loss inevitably happens during the operation, there is a high demand for differential control of blood, intracranial, and transmural pressure. Also, there are many unpredictable factors in an operation that regularly cause a high incidence of postoperative complications and, sometimes, aneurysms are not dealt with properly, which can lead to operative failure or cause sequelae such as epilepsy (Sandalcioglu et al., 2004; Molyneux et al., 2005; Fraser et al., 2006). Among patients with aneurysm rupture complicated with intracerebral hematoma who were treated by Sandalcioglu et al., the bleeding rate of aneurysm rupture was up to $30.8 \%$ during the operation (Sandalcioglu et al., 2004).

In our study, there were 13 elderly patients with Hunt and Hess grades ranging from grade 2 to 4 . All patients had multiple underlying diseases, and some were unable to tolerate drilling skull operations due to hematoma. We choose intravascular embolization for all patients with the permission of patient relatives and first treated those with aneurysms combined with internal carotid artery or vertebral artery stenosis. In these patients, when we performed aneurysm vascular embolization we conducted internal carotid artery or vertebral artery stent implantation. For patients with aneurysm combined with hematoma, we adopted mini-invasive aspiration. For patients with aneurysm combined with hydrocephalus, we adopted ventriculo-peritoneal drainage. Patients were treated comprehensively for 1-2 months postoperatively, all recovered well and had a good prognosis rate of up to $54 \%$, which is similar to the results of Ryttlefors et al. (Jain et al., 2004; Qureshi et al., 2007; Ryttlefors et al., 2008; Molyneux et al., 2009).

The combination of endovascular intervention plus surgery and mini-invasive aspiration avoids the possibility of damaging normal brain tissue during craniotomy, keeps intracranial pressure stable, and deals with the potential problem of a combined internal carotid artery. Vertebral artery stenosis occurred in most patients over the same period of time and this approach can help to decrease the incidence of complicating cerebral infarctions during and after operation. For this procedure, there is no specific limited condition and it can adapt to all types of aneurysms, especially for elderly patients (Yamashita et al., 1997). This approach improves prognoses, reduces the hospitalization period, and makes living conditions better. The small patient cohort represents the major shortcoming of this study. 


\section{REFERENCES}

Bracard S, Lebedinsky A, Anxionnat R, Neto JM, et al. (2002). Endovascular treatment of Hunt and Hess grade IV and V aneuryms. AJNR Am. J. Neuroradiol. 23: 953-957.

Bradac GB, Bergui M and Fontanella M (2005). Endovascular treatment of cerebral aneurysms in elderly patients. Neuroradiology 47: 938-941.

Donauer E, Reif J, al-Khalaf B, Mengedoht EF, et al. (1993). Intraventricular haemorrhage caused by aneurysms and angiomas. Acta Neurochir. 122: 23-31.

Fraser JF, Riina H, Mitra N, Gobin YP, et al. (2006). Treatment of ruptured intracranial aneurysms: looking to the past to register the future. Neurosurgery 59: 1157-1166.

Hijdra A, van Gijn J, Nagelkerke NJ, Vermeulen M, et al. (1988). Prediction of delayed cerebral ischemia, rebleeding, and outcome after aneurysmal subarachnoid hemorrhage. Stroke 19: 1250-1256.

Hodelin-Tablada R (1997). Spontaneous intracranial hematomas. A surgical solution? Rev. Neurol. 25: 1998-2002.

Jain R, Deveikis J and Thompson BG (2004). Endovascular management of poor-grade aneurysmal subarachnoid hemorrhage in the geriatric population. AJNR Am. J. Neuroradiol. 25: 596-600.

Linfante I and Wakhloo AK (2007). Brain aneurysms and arteriovenous malformations: advancements and emerging treatments in endovascular embolization. Stroke 38: 1411-1417.

Lubicz B, Leclerc X, Gauvrit JY, Lejeune JP, et al. (2004). Selective endovascular treatment of intracranial aneurysms with sapphire coils. AJNR Am. J. Neuroradiol. 25: 1368-1372.

Lubicz B, Piotin M, Mounayer C, Spelle L, et al. (2005). Selective endovascular treatment of intracranial aneurysms with a liquid embolic: a single-center experience in 39 patients with 41 aneurysms. AJNR Am. J. Neuroradiol. 26: 885-893.

Molyneux AJ, Kerr RS, Yu LM, Clarke M, et al. (2005). International subarachnoid aneurysm trial (ISAT) of neurosurgical clipping versus endovascular coiling in 2143 patients with ruptured intracranial aneurysms: a randomised comparison of effects on survival, dependency, seizures, rebleeding, subgroups, and aneurysm occlusion. Lancet 366: 809-817.

Molyneux AJ, Kerr RS, Birks J, Ramzi N, et al. (2009). Risk of recurrent subarachnoid haemorrhage, death, or dependence and standardised mortality ratios after clipping or coiling of an intracranial aneurysm in the International Subarachnoid Aneurysm Trial (ISAT): long-term follow-up. Lancet Neurol. 8: 427-433.

Qureshi AI, Janardhan V, Hanel RA and Lanzino G (2007). Comparison of endovascular and surgical treatments for intracranial aneurysms: an evidence-based review. Lancet Neurol. 6: 816-825.

Redekop GJ (2006). Microsurgical clipping or endovascular coiling for ruptured cerebral aneurysms. Stroke 37: 13521353.

Ryttlefors M, Enblad P, Kerr RS and Molyneux AJ (2008). International subarachnoid aneurysm trial of neurosurgical clipping versus endovascular coiling: subgroup analysis of 278 elderly patients. Stroke 39: 2720-2726.

Sandalcioglu IE, Schoch B, Regel JP, Wanke I, et al. (2004). Does intraoperative aneurysm rupture influence outcome? Analysis of 169 patients. Clin. Neurol. Neurosurg. 106: 88-92.

Shimoda M, Oda S, Mamata Y, Tsugane R, et al. (1997). Surgical indications in patients with an intracerebral hemorrhage due to ruptured middle cerebral artery aneurysm. J. Neurosurg. 87: 170-175.

Sugiu K, Tokunaga K, Watanabe K, Sasahara W, et al. (2005). Endovascular treatment for elderly patients with ruptured aneurysm. Acta Neurochir. Suppl. 94: 7-9.

Suzuki S, Jahan R, Duckwiler GR, Frazee J, et al. (2006). Contribution of endovascular therapy to the management of poor-grade aneurysmal subarachnoid hemorrhage: Clinical and angiographic outcomes. J. Neurosurg. 105: 664-670.

Wheelock B, Weir B, Watts R, Mohr G, et al. (1983). Timing of surgery for intracerebral hematomas due to aneurysm rupture. J. Neurosurg. 58: 476-481.

Yamashita K, Kashiwagi S, Kato S, Takasago T, et al. (1997). Cerebral aneurysms in the elderly in Yamaguchi, Japan. Analysis of the Yamaguchi Data Bank of Cerebral Aneurysm from 1985 to 1995. Stroke 28: 1926-1931. 